$740.6-711$

0>6 6

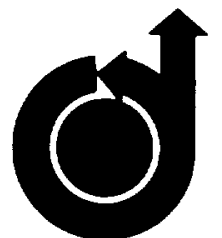

AIAA 80-1373R

Skin Friction Measurements by a Dual-Laser-Beam Interferometer Technique D. J. Monson and H. Higuchi 


\title{
Skin Friction Measurements by a Dual-Laser-Beam Interferometer Technique
}

\author{
D. J. Monson* \\ NASA Ames Research Center, Moffett Field, Calif. \\ and \\ H. Higuchi $\dagger$ \\ Dynamics Technology, Inc., Torrance, Calif.
}

\begin{abstract}
A portable dual-laser-beam interferometer that nonintrusively measures skin friction by monitoring the thickness change of an oil film subject to shear stress is described. The method is an advance over past versions in that the troublesome and error-introducing need to measure the distance to the oil leading edge and the starting time for the oil flow has been eliminated. The validity of the method was verified by measuring oil viscosity in the laboratory, and then using those results to measure skin friction beneath the turbulent boundary layer in a low speed wind tunnel. The dual-laser-beam skin friction measurements are compared with Preston tube measurements, with mean velocity profile data in a "law-of-the-wall" coordinate system, and with computations based on turbulent boundary-layer theory. Excellent agreement is found in all cases. (This validation and the aforementioned improvements appear to make the present form of the instrument usable to measure skin friction reliably and nonintrusively in a wide range of flow situations in which previous methods are not practical.)
\end{abstract}

\section{Nomenclature}

$C_{i} \quad=$ coefficient in Eqs. (22) and (25)

$c_{f} \quad=$ local skin friction coefficient, $\tau / q$

$\mathrm{d} p / \mathrm{d} x=$ external flow pressure gradient

$G \quad=$ oil viscosity function, see Eq. (A17)

$g \quad=$ gravitational acceleration

$H \quad=$ skin friction function, see Eq. (A5)

I $=$ incidence angle for interferometer flat

$i \quad=$ incidence angle for oil

$N \quad=$ fringe number, see Eq. (A3)

$n_{8} \quad=$ interferometer flat index of refraction

$n_{0} \quad=$ oil index of refraction

$q \quad=$ free-stream dynamic pressure

$R \quad$ = refraction angle for interferometer flat

$r \quad=$ refraction angle for oil

$T \quad=$ interferometer flat thickness, or temperature

$=$ time

$x \quad=$ distance from oil film leading edge

$x_{s} \quad=$ distance correction for sur face tension

$x^{*}, y^{*} \quad=$ Preston tube coordinates, see Ref. 6

$y=$ oil thickness

$\alpha \quad=$ initial oil film leading-edge slope

$\Delta N \quad=$ incremental change in fringe number

$\Delta t \quad=$ incremental change in time

$\Delta x \quad=$ beam spacing

$\delta$

$\epsilon$

$\theta$

$\lambda$

$=$ fixed oil sublayer thickness on a surface

$=$ pressure gradient and gravity correction parameter, see Eq. (A13)

= surface inclination from horizontal

$=$ laser wavelength

$=$ oil kinematic viscosity

$=$ oil density

$=$ local skin friction

Presented as Paper 80-1373 at the AIAA 13th Fluid and Plasma Dynamics Conference, Snowmass, Colo., July 14-16, 1980; submitted Aug. 19, 1980; revision received Feb. 13, 1981. This paper is declared a work of the U.S. Government and therefore is in the public domain.

- Research Scientist, Physical Sciences Branch. Member AIAA.

tResearch Scientist. Member AlAA.

Superscripts

( )' = corrected or "effective" value

() = average value

\section{Introduction}

$\mathbf{B}_{\mathrm{in}}$ ECAUSE of the importance of determining skin friction in turbulent boundary layers, there has been a continuing effort to develop reliable and practical methods for its measurement. Winter ${ }^{1}$ has reviewed the major methods developed to date. These include the floating-element balance; mean-velocity profile data, together with the Clauser chart; the Preston tube; and surface thin-film heat-transfer gages. Except for the floating-element balance technique, all of the methods are indirect because they are based on the wall similarity in turbulent boundary layers. Thus, each one generally has a limited range of application. The floatingelement balance method has critical gap and alignment problems, especially when subjected to a pressure gradient, and it is often too delicate and expensive for general use. The versatility of all of the methods is limited in that they either require permanent installation in a surface or they are intrusive in the flow.

Recently, Tanner and Blows ${ }^{2}$ and Tanner 3,4 described a new viscosity balance method that overcomes many of the limitations of the other techniques. Their method uses laser interferometric thickness measurements of oil films flowing on surfaces subject to shear stress and relates those measurements to the surface shear stress through a simple theory. Higher order effects-from surface tension, gravity, pressure or shear gradients, and three-dimensionality of the flow-are easily accounted for. The method has several important advantages. First, it is a direct method like the floating-element balance technique and does not require calibration in a known flow. In addition, the instrument is simple and inexpensive, is potentially very accurate, can be used in any type of steady flow, is easy to locate at various points on a surface, and is nonintrusive because the oil film is usually too thin to significantly affect the air flow. ${ }^{2}$

Despite these advantages, the method has not been widely adopted because of several practical difficulties in Tanner's original procedure. His method required accurate 


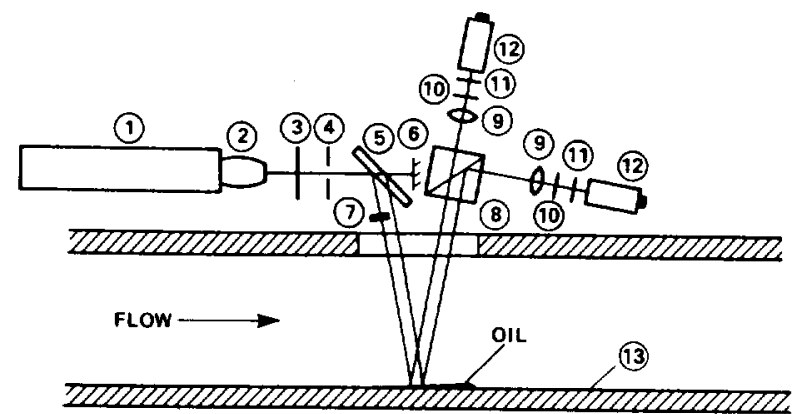
(1) HE-NE LASER
(2) TELESCOPE
(3) NEUTRAL DENSITY FILTER
(4) IRIS DIAPHRAGM
(5) INTERFEROMETER FLAT
(6) STOP
(7) HALF. WAVE RETARDATION PLATE

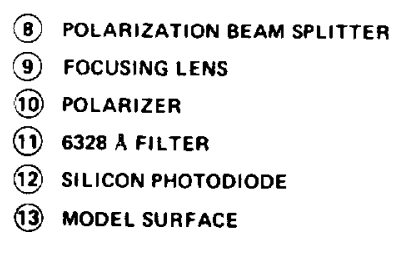

Fig. 1 Schematic of dual-laser-beam skin friction interferometer.

measurements of both the distance from the beam focal point to the oil film leading edge and of the time at which the oil flow started. Tanner ${ }^{3}$ measured the leading-edge distance before a run by visually setting the focal point of a reference beam with known spacing from the measurement beam at the oil leading edge. This can be subject to error in some applications because of the difficulty in exactly locating the oil edge and in accounting for oil spreading after the measurement. In addition, to measure the distance, Tanner found it necessary to rigidly mount his equipment to the windtunnel top wall; at that location, tunnel vibration and wall displacements can disturb the measurement, making beam repositioning difficult. Furthermore, Tanner assumed that the oil-flow time was equal to the tunnel running time. This also was subject to error because of prerun oil flow, tunnel starting transients, and early-run oil surface waves.

In this paper, a dual-laser-beam instrument with fixed beam spacing, and the procedures for its use are described. This method of measuring skin friction eliminates most of the limitations of Tanner's technique. The need to measure the distance to the oil leading edge has been eliminated. Further, the theory has been extended to determine the "effective" oilflow time from the interference fringe count as a function of time, rather than by direct measurement. This automatically eliminates all of the inherent timing errors in the original method. Finally, all of the instrument components are located remotely from the wind tunnel. This eliminates nearly all effects of tunnel vibrations, makes relocating the measurement point on the surface easy, and leads to an instrument that is easily transportable from one tunnel to another.

\section{Description of Instrument and Theory}

A typical wind tunnel installation of the dual-laser-beam instrument is shown in Fig. 1. The instrument measures the rate of change in thickness of a flowing oil film at two points just behind the leading edge of the film. The linearly polarized output from a He-Ne laser is expanded by a telescope and passed through a neutral density filter to reduce the beam power level to a value that avoids excessive oil heating (see discussion by Tanner ${ }^{3}$ ). The single beam is then divided into two parallel beams, using an interferometer flat. One beam passes through a half-wave retardation plate to rotate its polarization by $90 \mathrm{deg}$, and both beams are focused on the oil. The beams reflect off the model surface, are then separated with a polarization beam splitter, and each is focused on a photodiode.

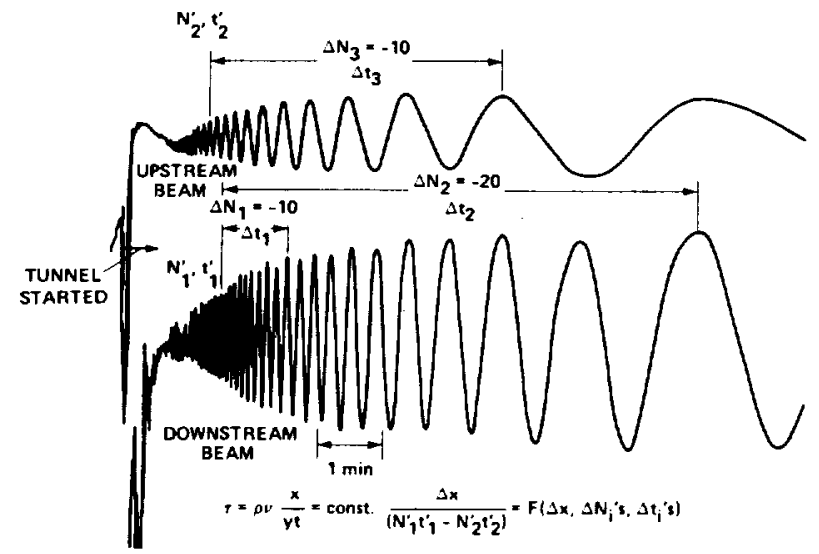

Fig. 2 Typical dual-laser-beam interferometer fringe record from a wind-tunnel test; beam spacing is $5 \mathrm{~mm}$.

Polarizers and narrow-band interference filters are used at the detectors for additional noise reduction. The detector signals are recorded on a two-channel chart recorder. As the oil thickness changes, the recorded light intensity is modulated by alternating constructive and destructive interference between beams reflected from the oil and model surfaces. ${ }^{2}$ The transmitting and receiving optics are mounted on tripods separate from the wind tunnel for versatility in locating the measurement points.

A typical dual-beam interferometer output record is shown in Fig. 2. Each crest on a trace represents an oil thickness corresponding to constructive interference between the reflections. Thus, by simply counting the number of fringes over a given time span, the resulting change in oil thickness can be precisely computed in terms of the known laser wavelength. The erratic behavior of the signals just after the tunnel is started is due to transient waves observed in the oil. Usable traces begin after the waves disappear and after the oil thins to the point where only a single fringe occurs over the diameter of the laser focal spot diameter.

The procedure for computing skin friction from interferometer records such as those shown in Fig. 2 is given in the Appendix. The oil density, index of refraction, spacing between the two laser measurement beams, and the laser wavelength are constants that are required as input for the calculation. Also, the oil kinematic viscosity must be accurately known over the test temperature range. If needed, this can be easily measured using the dual-beam interferometer from a gravity flow experiment and the reduction procedure (also given in the Appendix). An advantage of measuring the oil viscosity in this way is that the laser beam spacing does not have to be known-as can be seen from Eqs. (A5) and (A21) of the Appendix, it cancels out of the final expression for skin friction. Finally, the laser incidence angle with respect to the test surface must be measured, although if it is close to $90 \mathrm{deg}$, high accuracy is not required.

\section{Description of Experiment}

\section{Oil Viscosity Measurement}

The present dual-beam instrument was first used in a gravitational flow setup to measure the viscosity of several Dow Corning 200 silicon oils. As pointed out by Tanner ${ }^{4}$ silicon oil is desirable for skin friction measurements because it is available in a wide range of viscosities that are relatively insensitive to temperature; moreover, it has low surface tension and a very low vapor pressure. The viscosity measurements were made on a polished aluminum disk, on the back of which a thermocouple was mounted to measure oil temperature. A timing mark was recorded when the disk 
Fig. 3 The $1 \mathrm{ft} \times 1 \mathrm{ft}$ low-speed wind tunnel.

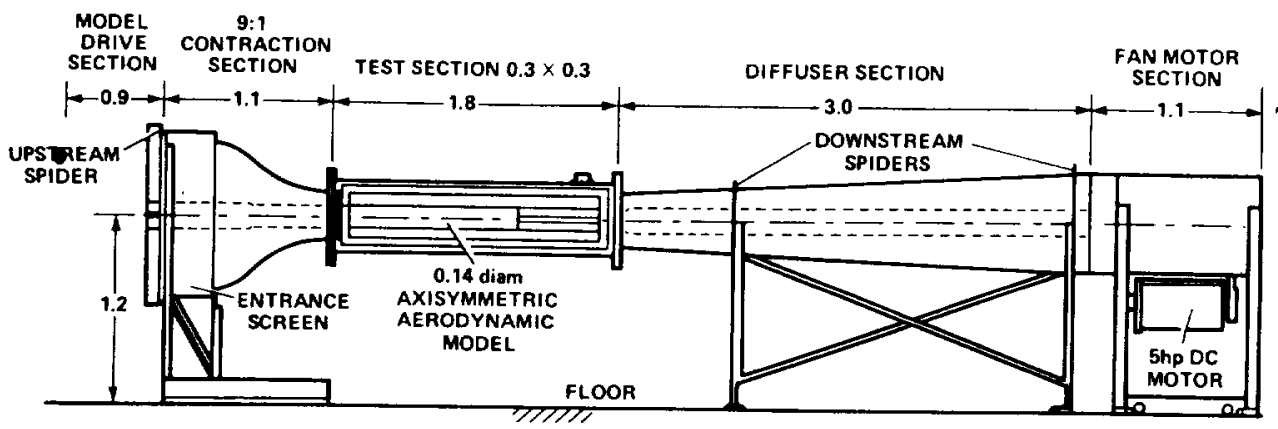

DIMENSIONS IN m

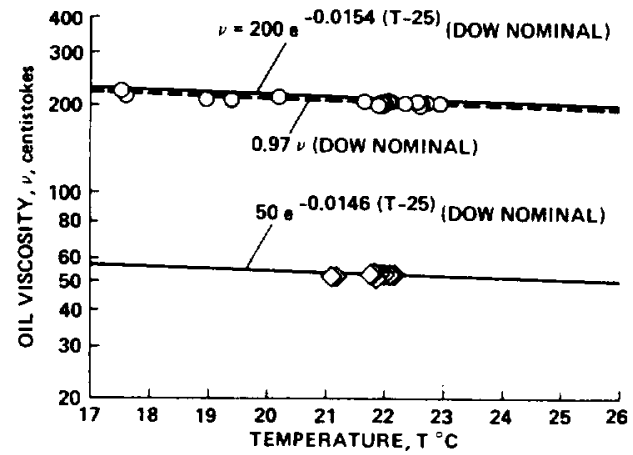

Fig. 4 Silicon oil viscosities measured with the dual-laser-beam interferometer.

was tipped from a horizontal to a vertical position to initiate the flow. Results of the viscosity measurements are given in the next section.

\section{Skin Friction Measurement}

Skin friction measurements with the dual-beam interferometer were made in the test facility shown in Fig. 3. The facility is an open-cycle, low-speed wind tunnel with a 14$\mathrm{cm}$-diam aluminum cylinder extending along the tunnel axis from the tunnel entrance to the diffuser. The maximum freestream speed is $50 \mathrm{~m} / \mathrm{s}$. A fully developed turbulent boundary layer exists on the cylinder. The skin friction measurements were made at three axial stations on the cylinder at a speed of $37 \mathrm{~m} / \mathrm{s}$, and at the central station over a speed range of 21 to $48 \mathrm{~m} / \mathrm{s}$. Large plexiglass side windows allowed laser beam access in and out of the tunnel. The oil temperature was measured by inserting a thermocouple in a metal plug in the tunnel wall. After the laser beams were located and aligned, oil was placed along a line just upstream of the forward beam reflection point. When the tunnel was started and the oil flow initiated, a timing mark was recorded and the measurements were initiated, as shown in Fig. 2.

For purposes of validation, a simultaneous measurement of skin friction was obtained with a $1.07-\mathrm{mm}$-diam Preston tube; a measurement was also derived from mean velocity profiles. The Preston tube measurements were made at the same three axial stations as the oil-flow measurements, as well as at several azimuthal locations for each station to verify symmetry of the tunnel flow. The mean velocity profiles were taken only on the top of the cylinder at the central station and only at freestream speeds of 22 and $36 \mathrm{~m} / \mathrm{s}$. Skin friction was computed from the Preston tube data, using the procedures of Patel. ${ }^{5}$ The Patel calibration consists of a set of three calibration curves expressed in terms of the Preston tube coordinates, $x^{*}$ and $y^{*}$ [see Eqs. (2-4) of Ref. 5]. These, however, do not match at the crossover points that were suggested by Patel and thus give unnatural discontinuities in the computed skin friction. Therefore, in this paper, the intersections of the three calibration curves were numerically

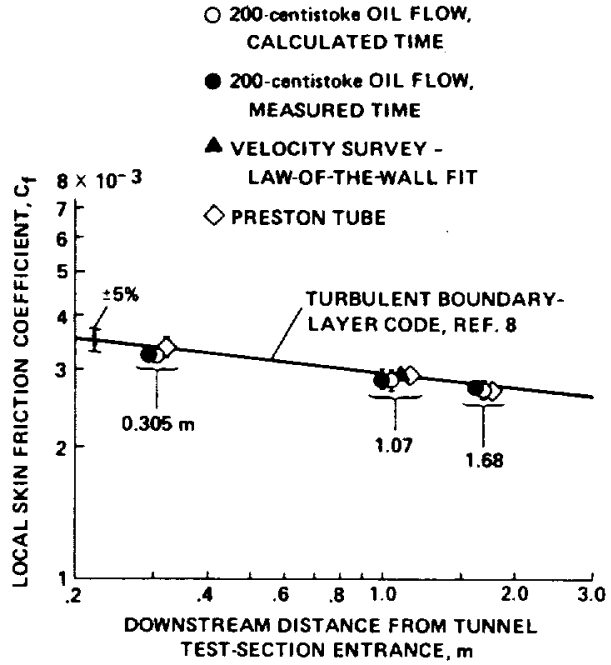

Fig. 5 Comparison of skin friction measurements at $37 \mathrm{~m} / \mathrm{s}$. Note: The data from each station are spread horizontally for visibility; all correspond to the same location.

obtained and applied in the data reduction to eliminate the discontinuities.

Skin friction from the mean velocity profiles was obtained by plotting the data on a Clauser chart to fit the law-of-thewall, as suggested by Coles. ${ }^{6}$ Although the boundary layers were axisymmetric, no transverse curvature effect was observed on these plots.

\section{Results and Discussions}

Results of the measurements of Dow silicon oil viscosity using the dual-beam interferometer are shown in Fig. 4 for oils of nominally 50 - and $200-\mathrm{cS}$ viscosity. The data are compared with nominal curves from Dow's product literature. The data were reduced using computed oil-flow times by the method described in the Appendix. The 50-cS oil data lie within $\pm 2 \%$ of the Dow curve, and the $200-\mathrm{cS}$ oil data lie within $\pm 2 \%$ of a curve that is $97 \%$ of the Dow curve. These variations are within the supplier's tolerances and those by Tanner. ${ }^{7}$ Both curves on Fig. 4 were used to obtain the oil viscosity at a given temperature for the skin friction calculations.

Measurements of skin friction using 200-cS oil, a boundarylayer velocity survey, and Preston tube measurements are compared in Fig. 5. The data were measured at three axial locations on the cylinder at a freestream speed of $37 \mathrm{~m} / \mathrm{s}$. They are also compared with an axisymmetric turbulent boundary-layer code that incorporated the Wilcox-Rubesin Reynolds stress equation turbulence model. ${ }^{8}$ As an initial condition for computation, the momentum thickness in the code was matched with the measured momentum thickness at the tunnel test section entrance. Higuchi and Rubesin ${ }^{9}$ found that this turbulence model and matching condition gave good 


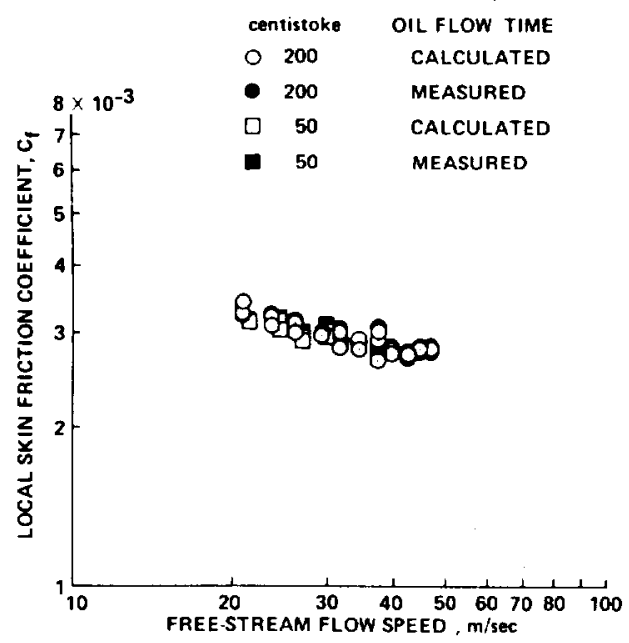

Fig. 6 Oil-flow skin friction measurements $1.07 \mathrm{~m}$ from the tunnel test-section entrance.

agreement with measured skin friction on an axisymmetric model similar to that in the present tests. The dual-beam interferometer data were reduced, using both measured and calculated flow times for comparison. The error bars shown represent the maximum scatter measured for several repeated runs. The Preston tube data include various azimuthal positions on the axisymmetric cylinder to check for flow symmetry.

The dual-beam interferometer skin friction results shown in Fig. 5 are in excellent agreement with both the Preston tube results at all three locations and with the result from the velocity profile at the central station. The fairly large scatter in the Preston tube data at the forward station arises from flow asymmetry caused by the tunnel entrance screen. All measurements agree well with the turbulent boundary-layer calculation at the two downstream stations. The dual-beam interferometer data at the forward station are a few percentage points lower than predicted, possibly because of the aforementioned flow asymmetry. Finally, notice that the dual-beam interferometer results are identical, using either measured or calculated flow times.

As an alternative test of the dual-beam interferometer skin friction technique, additional measurements were made at the central axial station over a wide range of freestream flow speed. The results for both $50-$ and $200-\mathrm{cS}$ viscosity oil are shown in Fig. 6. The less viscous 50-cS oil was used only at lower speeds. The scatter in the data represents the maximum variation for repeated runs at each flow speed. Within this scatter, the 50-cS dual-beam interferometer data are in excellent agreement with the 200 -cS results. This agreement for a factor of 4 change in oil viscosity provides increased confidence in the method and demonstrates that the agreement of the $200-\mathrm{cS}$ oil data with other methods shown in Fig. 5 is not fortuitous. Finally, notice that the results are again identical, using either measured or calculated flow times.

Some of the dual-beam interferometer results shown in Fig. 6 are compared in Fig. 7 with Preston tube and boundarylayer velocity survey measurements, and with the turbulent boundary-layer prediction. The dual-beam interferometer data are for both oil viscosities, but using only calculated flow times. The error bars represent the maximum scatter measured for repeated runs. For the Preston tube at $21 \mathrm{~m} / \mathrm{s}$, measurements were made at various azimuthal locations. Within this scatter and the accuracy of the measurements, the dual-beam interferometer data are again in excellent agreement with the Preston tube data over the entire speed range, and also with the two velocity survey results at 22 and $36 \mathrm{~m} / \mathrm{s}$. All methods disagree only slightly with the turbulent boundary-layer prediction, although the measured data appear slightly higher than the prediction at the lowest speeds.
O 50 AND 200 centistoke OIL FLOW

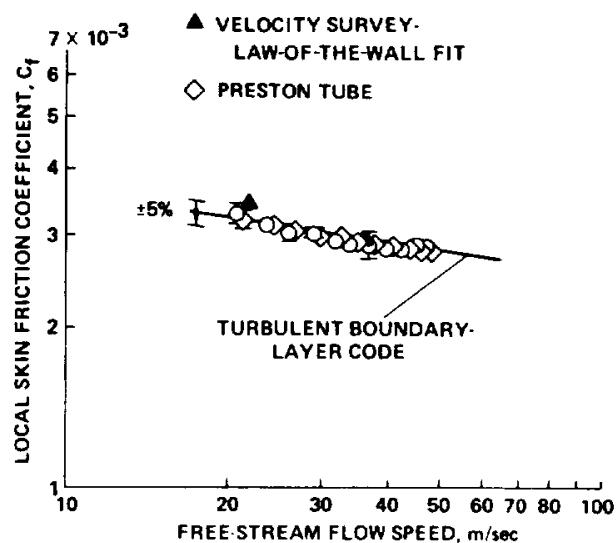

Fig. 7 Comparison of skin friction measurements $1.07 \mathrm{~m}$ from the tunnel test-section entrance.

Part of this discrepancy could be caused by the previously discussed flow asymmetry. Overall, the measurements for all three methods and the prediction are within $5 \%$ of each other.

A major goal of this study was to verify the present method in which the oil film leading-edge distance and the flow duration are computed rather than measured. To test the method, the leading-edge distances were directly measured in the gravity flow tests using the method discussed by Tanner, ${ }^{3}$ and the oil-flow durations were measured in all of the tests. The flow durations in this study were deliberately chosen to be fairly long; this was done to minimize the influence on the described measurements of any transient events, at the start of the oil flows, that might introduce errors. (The oil viscosity tests were recorded for 20-60 min, and the skin friction tests for 2-10 min, depending on oil viscosity and air speed.) Although not required for the dual-beam oil viscosity measurements, the oil-film leading-edge distances can be computed from Eq. (A20) in the Appendix.

The computed leading-edge distances for the oil viscosity tests in this study ranged between 93 and $100 \%$ of the measured values. The slightly lower computed values are consistent with the effect of surface tension on this type of flow, as discussed in the Appendix. The computed flow-times ranged between 97 and $103 \%$ of the measured values for the gravity flow tests, and between 98 and $106 \%$ for the skin friction tests. For the latter, the slightly higher computed flow durations are consistent with the initial presence of surface waves in the flow, which add to the rate of oil removal. This close agreement explains why the skin friction results are the same in Figs. 5 and 6, using either measured or computed flow times. The above comparisons, plus the previously discussed good agreement between the dual-beam interferometer skin friction results and other methods, provide solid confirmation of the method presented in the Appendix.

The only problem encountered in the operation of the new dual-beam interferometer arose from occasional dust particles in the oil. The wind tunnel had no inlet filter and was located in a fairly dusty area with high daytime activity. Many dust particles could be observed on the oil surface after most runs. They could cause two problems: 1) a dust particle exactly at a beam focal point surely could cause erratic interferometer behavior; and 2) a large dust particle just ahead of a beam focal point could locally perturb either the oil or the air flow enough to influence the skin friction measurement, but could not be detectable on the interferometer records. In fact, the latter was probably the largest cause of the oil-flow data scatter found in this study. Support for this view is found from the data in Fig. 5. The measurements at the forward station were taken at a time when there was less dust than normal in the air, and the scatter there is much less than for the other measurements. 


\section{Conclusions}

A dual-laser-beam interferometer for measuring skin friction in wind tunnels by monitoring the thickness change of an oil film at two points has been described. The accuracy of the present method has been well established by extensive comparisons with results of conventional measurements and with predictions based on turbulent boundary-layer theory, at least for the low-speed two-dimensional turbulent boundary layer studied here. (Recent tests have successfully applied the method in supersonic flow as well. That work will be described in a future publication.) The method that is developed to compute the required oil-film flow time and leading-edge distance from the interferometer records has been verified. The operational problems with the method have been shown to be minimal, with the possible exception of that related to dust in the flow. The method appears to be widely applicable for the nonintrusive measurement of skin friction in many complex flows where other techniques are limited or impractical.

\section{Appendix}

\section{Skin Friction Theory}

Tanner and Blows ${ }^{2}$ show that an oil film on a surface subject to a constant shear stress will assume the linear shape

$$
x=\tau y t / \rho \nu
$$

(This equation is exact only for constant shear stress. But as Tanner and Blows ${ }^{2}$ discuss, it will hold with good accuracy near the leading edge of an oil film, whatever the shear stress distribution, as long as the percentage change in shear over the distance from the leading edge to the measurement point is small. The appropriate technique would then be to deposit oil droplets at points of interest and to determine $\tau$ for the position of each droplet by applying the above equation. The technique can also be extended to large shear gradients by solving an integral for $\tau$ over $x$ (see Ref. 2), but that is not done in this report.) Tanner ${ }^{3,4}$ observed that a very small layer of oil, with thickness $\delta$ of the order of a few nanometers, will always stick to a surface without flowing. He also introduced a finite initial leading-edge slope $\alpha$ to approximately account for any prerun oil flow. Tanner ${ }^{2}$ showed by computation that the effect of leading-edge surface tension is unimportant for the case of skin friction acting on the oil. Taking these effects into account, Eq. (A1) may be written as:

$$
x=\frac{\tau(y-\delta) t}{\rho \nu}+\alpha^{-1}(y-\delta)
$$

To relate the oil thickness to a laser interferometer beam with wavelength $\lambda$, the quantity $y$ can be expressed in terms of the fringe number $N$ by

$$
N \lambda=2 n_{0} y \operatorname{coc}(r)
$$

where

$$
\cos (r)=\cos \left[\arcsin \left(\frac{\sin (i)}{n_{0}}\right)\right]
$$

By this definition, $N$ will assume integer values for oil thicknesses corresponding to reflected beam constructive interference. Combining Eqs. (A2) and (A3), and defining

$H=\frac{\tau \lambda}{2 n_{0} \rho \nu \cos (r)}, N^{\prime}=N-\frac{2 n_{0} \delta \cos (r)}{\lambda}, t^{\prime}=t+\rho \nu / \alpha \tau$

Eq. (A2) becomes

$$
x=H N^{\prime} t^{\prime}
$$

In Eq. (A6), $N^{\prime}$ and $t^{\prime}$ are now defined as the effective fringe number and oil flow time, respectively. They differ from the original variables by unknown constants. Notice, however, that the incremental change is the same for either effective or original variables. Furthermore, the effective fringe number will not necessarily assume integer values at the crests of the interferometer traces as the original fringe number does.

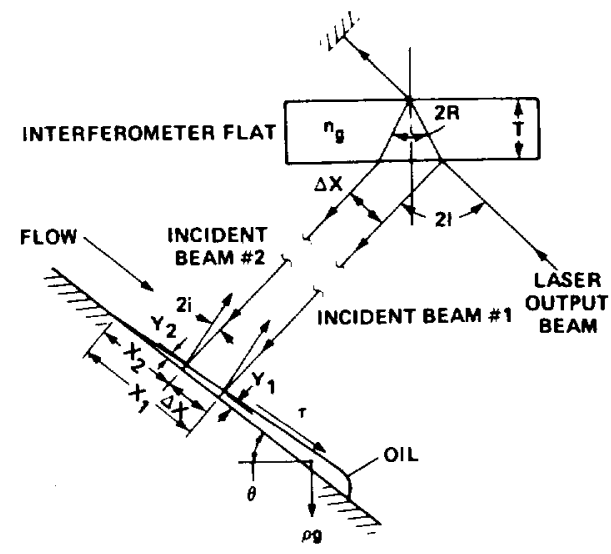

Fig. A1 Geometry and notation for dual-laser-beam interferometer oil film measurement.

The geometry and notation for the dual-laser-beam oil-film measurement is shown in Fig. Al. Using this and the notation of Fig. 2, a difference form of Eq. (A6) may be written as

$$
H=\frac{\Delta x}{\Delta\left(N^{\prime} t^{\prime}\right)}=\frac{\Delta x}{\left(N_{j}^{\prime} t_{l}^{\prime}-N_{2}^{\prime} t_{2}^{\prime}\right)}
$$

This equation forms the basis for the dual-beam interferometer with known beam spacing, $\Delta x$.

To solve for the fringe numbers $\left(N^{\prime}\right)$ and oil flow times $\left(t^{\prime}\right)$ in Eq. (A7), notice from Eq. (A6) that the product $N^{\prime} t^{\prime}$ is constant at each beam location, because $x$ and $H$ also are constant. Thus, equating the product $N^{\prime} t^{\prime}$ at different arbitrary times on each interferometer trace, one obtains (in the notation of Fig. 2)

$$
\begin{gathered}
N_{1}^{\prime}=-\Delta N_{1} \frac{\Delta N_{2}}{\Delta N_{1}}\left(\frac{\Delta t_{2}}{\Delta t_{1}}-1\right) /\left(\frac{\Delta t_{2}}{\Delta t_{1}}-\frac{\Delta N_{2}}{\Delta N_{1}}\right) \\
t_{1}^{\prime}=-\Delta t_{1}\left(\frac{N_{1}^{\prime}}{\Delta N_{1}}+1\right) \\
N_{2}^{\prime}=-\Delta N_{3}\left(\frac{t_{2}^{\prime}}{\Delta t_{3}}+1\right)
\end{gathered}
$$

Note that, although Fig. 2 illustrates beam one as the downstream beam, this choice is arbitrary. Also note that once the time $t_{1}^{\prime}$ is computed from Eq. (A9), this immediately makes the time of any other point on either fringe record known by measuring from $t_{i}^{\prime}$ the incremental change forward or backward to that point on the chart record. The time $t_{2}^{\prime}$ which appears in Eq. (A10) is computed in this manner.

In this study, the time intervals are measured from the crests of the fringe traces, as shown in Fig. 2, although the choice is arbitrary. Tanner ${ }^{3}$ measured time from the trace zero-crossing points, but that would work well only when there is no drift in the average signal level with time. Also, the arbitrary choice was made in this study to compute the effective flow time from the downstream beam record because of the larger number of fringes that occurred there.

The beam spacing $\Delta x$ can be either measured or computed from geometric optics as (see Fig. A1 for notation)

$$
\Delta x=2 T \cos (I) \tan (R)
$$

where

$$
\tan (R)=\tan \left[\arcsin \left(\sin (I) / n_{g}\right)\right]
$$

In the above equations, notice that $\Delta x$ can be arbitrarily chosen by tilting the beam-splitting flat. With $\Delta x$ known, Eq. (A7) is solved for $H$, and finally, Eq. (A5) is solved for $\tau$.

If an external flow pressure gradient or gravity force is acting on the oil together with shear stress, as shown in Fig. A1, no simple oil-shape solution is possible. However, Tanner and Blows ${ }^{2}$ derived an approximate expression to account for these forces as long as their effect is small compared to the effect of the shear stress. They define a small perturbation 
parameter $\epsilon$, such that

$$
\tau^{\prime}=(1-\epsilon)^{-1} \tau
$$

where

$$
\epsilon=\left[\frac{1}{3} \frac{y}{\tau}+\frac{2}{3} \frac{\rho \nu x}{\tau^{2} t}\right]\left[\frac{\mathrm{d} p}{\mathrm{~d} x}-\rho g \sin (\theta)\right]
$$

To further simplify Eq. (A13), assume that the shear stress within the bracket is the uncorrected value, set $y$ equal to $y_{1}$, eliminate $x$ through $\mathrm{Eq}$. (A1), and introduce the fringe number through Eq. (A3). The result is:

$$
\epsilon \cong \frac{\lambda N_{i}^{\prime}}{2 n_{0} \tau \cos (r)}\left[\frac{\mathrm{d} p}{\mathrm{~d} x}-\rho g \sin (\theta)\right]
$$

Notice that $\epsilon$ can be made arbitrarily small by allowing the oil to thin, thus reducing $N_{l}^{\prime}$. The parameter $\epsilon$ was zero for the present study. However, introducing typical values from this study into Eq. (A14) and assuming a vertical surface gives values for $\epsilon$ between $2 \times 10^{-2}$ and $3 \times 10^{-2}$. Thus, at least for similar conditions, the small perturbation analysis of Tanner and Blows is justified.

\section{Oil Viscosity Theory}

With gravity as the only force, Tanner and Blows ${ }^{2}$ show that an oil film will assume the parabolic shape

$$
x=y^{2} \operatorname{tg} \sin (\theta) / \nu
$$

Introducing $\delta$ and $\alpha$ as before, and introducing a correction to $x$ for the effect of surface tension near the film leading edge, ${ }^{7}$ $x_{s}$, results in Eq. (A15) becoming

$$
x=\left[(y-\delta)^{2} \operatorname{tg} \sin (\theta) / \nu\right]+\alpha^{-1}(y-\delta)+x_{s}
$$

Then, obtaining the fringe number from $\mathrm{Eq}$. (A3) and using the definition of effective fringe number appearing in Eq. (A5), we define

$$
G=\lambda^{2} g \sin (\theta) / 4 v n_{0}^{2} \cos ^{2}(r)
$$

Equation (A16) then becomes

$$
x-x_{s}=G N^{\prime 2}\left[t+\frac{2 \nu n_{0} \cos (r)}{\alpha \lambda g \sin (\theta) N^{\prime}}\right]
$$

Unlike the previous analysis for shear stress, notice that the introduction of $\alpha$ here results in a variable term containing $N^{\prime}$ rather than a constant term added to the time. Tanner' also derived an expression for $x_{s}$ and found it to depend on $N^{\prime}$. However, Tanner ${ }^{3.7}$ estimated that after "reasonable" oilflow times, $x_{5}$ and the term containing $\alpha$ are both quite small compared to $x$ and $t$, respectively. Thus, good accuracy would be retained by neglecting these small-terms in Eq. (A18) altogether. However, a more general analysis can be made by assuming that the incremental changes in $N^{\prime}$ for any set of measurements from interferometer records are small compared to $N^{\prime}$ itself. (This can be ensured by taking the measurements over a small enough time interval.) Then, an effective distance and time can be defined, respectively, as

$$
x^{\prime} \cong x-\bar{x}_{s}, \quad t^{\prime} \cong t+\frac{2 v n_{0} \cos (r)}{\alpha \lambda g \sin (\theta) \bar{N}^{\prime}}
$$

where $\bar{x}_{s}$ and $\bar{N}^{\prime}$ are average values during a measurement time interval. With these definitions, Eq. (A18) becomes

$$
x^{\prime}=G N^{\prime 2} t^{\prime}
$$

and a difference version may be written as

$$
G=\frac{\Delta x}{\Delta\left(N^{\prime 2} t^{\prime}\right)}=\frac{\Delta x}{\left(N_{l}^{\prime 2} t_{l}^{\prime}-N_{2}^{\prime 2} t_{2}^{\prime}\right)}
$$

Equation (A21) forms the basis of the dual-beam interferometer measurement of oil viscosity. Notice that $\bar{x}_{s}$ is eliminated from this equation.

To solve for the $N^{\prime} s$ and $t^{\prime} s$, we can take advantage of the fact shown in Eq. (A20) that the product $N^{\prime}{ }^{2} t$ is constant for fixed $x^{\prime}$. In an analysis similar to the one for shear stress, this leads to a cubic equation for $N_{i}^{\prime}$ as

$$
N_{3}^{\prime 3}+\frac{C_{2}}{C_{1}} N_{1}^{\prime 2}+\frac{C_{3}}{C_{1}} N_{1}^{\prime}+\frac{C_{4}}{C_{1}}=0
$$

where

$$
\begin{aligned}
& C_{1}=2\left[\frac{\Delta t_{2}}{\Delta t_{1}}-\frac{\Delta N_{2}}{\Delta N_{1}}\right] \\
& C_{2}=\Delta N_{1}\left[4 \frac{\Delta N_{2}}{\Delta N_{1}}\left(\frac{\Delta t_{2}}{\Delta t_{1}}-1\right)+\frac{\Delta t_{2}}{\Delta t_{1}}-\left(\frac{\Delta N_{2}}{\Delta N_{1}}\right)^{2}\right] \\
& C_{3}=2 \Delta N_{1} \Delta N_{2}\left[\left(\frac{\Delta N_{2}}{\Delta N_{1}}+I\right)\left(\frac{\Delta t_{2}}{\Delta t_{1}}-I\right)\right] \\
& C_{4}=\Delta N_{1}\left(\Delta N_{2}\right)^{2}\left[\frac{\Delta t_{2}}{\Delta t_{1}}-I\right]
\end{aligned}
$$

Also,

$$
t_{l}^{\prime}=-\Delta t_{1}\left[\left(\frac{N_{1}^{\prime}}{\Delta N_{1}}+1\right)^{2} /\left(2 \frac{N_{1}^{\prime}}{\Delta N_{1}}+1\right)\right]
$$

and

$$
N_{2}^{\prime 2}+C_{5} N_{2}^{\prime}+C_{6}=0
$$

where

and

$$
C_{5}=2 \Delta N_{3}\left[\left(t_{2}^{\prime} / \Delta t_{3}\right)+1\right]
$$

$$
C_{6}=\left(\Delta N_{3}\right)^{2}\left[\left(t_{2}^{\prime} / \Delta t_{3}\right)+I\right]
$$

Finally, the oil viscosity is computed from Eq. (A27), which is the result of combining Eqs. (A21) and (A17), and of knowing $\Delta x$.

$$
\nu=\frac{\lambda^{2} g \sin (\theta)}{4 n_{0}^{2} \cos ^{2}(r)}\left[\frac{\left(N_{1}^{\prime 2} t_{1}-N_{2}^{\prime 2} t_{2}^{\prime}\right)}{\Delta x}\right]
$$

\section{References}

${ }^{1}$ Winter, K. G., "An Outline of the Techniques for the Measurement of Skin Friction in Turbulent Boundary Layers," Progress in the Aerospace Sciences, Vol. 18, Pergamon Press, Great Britain, 1977, pp. 1-57.

${ }^{2}$ Tanner, L. H. and Blows, L. G., "A Study of the Motion of Oil Films on Surfaces in Air Flow, with Application to the Measurement of Skin Friction," Journal of Physics E: Scientific Instruments, Vol. 9, March 1976, pp. 194-202.

${ }^{3}$ Tanner, L. H., "A Skin Friction Meter, Using the Viscosity Balance Principle, Suitable for Use with Flat or Curved Metal Surfaces," Journal of Physics E: Scientific Instruments, Vol. 10, March 1977, pp. 278-284.

"Tanner, L. H., "A Comparison of the Viscosity Balance and Preston Tube Methods of Skin Friction Measurement," Journal of Physics E: Sciensific Instruments, Vol. 10, June 1977, pp. 627-632.

${ }^{5}$ Patel, V. C., "Calibration of the Preston Tube and Limitations on Its Use in Pressure Gradients," Journal of Fluid Mechanics, Vol. 23, Pt. 1, Sept. 1965, pp. 185-205.

'Coles, D., "The Young Person's Guide to the Data," Proceedings AFOSR-IFP-Stanford Conference on Computation of Turbulent Boundary Layers, Vols. I and II, edited by S. J. Kline, et al., Dept. of Mechanical Engineering, Stanford University, Aug. 1968.

${ }^{7}$ Tanner, L. H., "Two Accurate Optical Methods for Newtonian Viscosity Measurements, and Observations on a Surface Effect with Silicon Oil," Journal of Physics E: Scientific Instruments, Vol. 10, Oct. 1977, pp. 1019-1028.

${ }^{8}$ Wilcox, D. C. and Rubesin, M. W., "Progress in Turbulence Modeling for Complex Flowfields, Including Effects of Compressibility," NASA TP-1517, April 1980.

${ }^{9}$ Higuchi, H. and Rubesin, M. W., "Behavior of a Turbulent Boundary Layer Subjected to Sudden Transverse Strain," AIAA Journal, Vol. 17, Sept. 1979, pp. 931-941. 